Enfermagem Brasil 2018;17(6):694-701

https://doi.org/10.33233/eb.v17i6.1306

\title{
REVISÃO \\ Complicações da intervenção coronária percutânea: inferência dos diagnósticos de enfermagem
}

\author{
Eduardo Tavares Gomes, M.Sc. *, Tatiane Teodósio da Silva**, Marcileide da Silva Santos**
}

*Enfermeiro, Doutorando em Ciências pela Escola de Enfermagem da Universidade Federal de Pernambuco, ${ }^{* *}$ Enfermeira, graduada pela Faculdade de Enfermagem UNINASSAU

Recebido em 3 de novembro de 2017; aceito em 13 de novembro de 2018.

Endereço de correspondência: Eduardo Tavares Gomes, Hospital das Clínicas da Universidade de Federal Pernambuco, Avenida Profo Moraes Rego, 1235, Cidade Universitária, 50670-901 Recife PE, E-mail: edutgs@hotmail.com; Tatiane Teodósio da Silva: tati_enf@hotmail.com, Marcileide da Silva Santos: marcicleide_santos@hotmail.com

\section{Resumo}

Objetivos: Realizar a inferência de diagnósticos de enfermagem (DE) aplicáveis às principais complicações das intervenções coronárias percutâneas (ICP). Métodos: Revisão da literatura a partir da qual foi realizada a inferência dos DE aplicáveis nas principais complicações das ICP. $\mathrm{Na}$ etapa da revisão integrativa foram buscados artigos nas bases Lilacs, BDEnf e Scielo, com o descritor "angioplastia", que abordasse as complicações relacionadas ao procedimento. Após a avaliação das complicações, foram realizadas as inferências dos DE aplicáveis. Resultados: Treze artigos compuseram o corpus de análise. Os principais DE aplicáveis inferidos são: Eliminação urinária prejudicada; Padrão respiratório ineficaz; Risco de perfusão tissular cardíaca diminuída; Perfusão tissular periférica ineficaz; Risco de perfusão tissular cerebral ineficaz; Débito cardíaco diminuído; Confusão aguda; Integridade da pele prejudicada; Integridade tissular prejudicada. Conclusão: As taxonomias de enfermagem permitem DE aplicáveis que norteiem a sistematização da assistência nas complicações das ICP.

Palavras-chave: angioplastia, enfermagem cardiovascular, diagnóstico de enfermagem, cardiologia.

\begin{abstract}
Complications of percutaneous coronary intervention: inference of nursing diagnostics

Objective: To make an inference of nursing diagnosis (ND) applicable in the main complications of percutaneous coronary interventions (PCl). Methods: Literature review from which the inference of applicable ND was conducted in major complications of $\mathrm{PCl}$. Integrative review articles were searched in Lilacs, BDEnf and Scielo with angioplasty descriptor that addressed the complications related to the procedure. After evaluation of complications, inference of ND applicable was made. Results: Thirteen articles were included in the analysis corpus. The main ND inferred applicable are: impaired urinary elimination; Ineffective breathing pattern; Decreased heart tissue perfusion risk; Ineffective peripheral tissue perfusion; Ineffective cerebral tissue perfusion risk; Decreased cardiac output; Acute confusion; Impaired skin integrity; Impaired tissue integrity. Conclusion: Taxonomies nursing allow ND applicable to guide the systematization of assistance on the complications of $\mathrm{PCl}$.
\end{abstract}

Key-words: angioplasty, cardiovascular nursing, nursing diagnosis, cardiology.

\section{Resumen \\ Complicaciones de intervención coronaria percutánea: inferencia de los diagnósticos de enfermería}

Objetivo: Evaluar la literatura sobre las principales complicaciones de las intervenciones coronarias percutáneas (ICP) y realizar la inferencia de los diagnósticos de enfermería (DE) aplicables en estos casos. Métodos: Estudio metodológico en el que se realizó la inferencia de los DE aplicables en las principales complicaciones de las ICP a partir de la revisión integradora de la literatura sobre el tema. En la revisión integral, se realizaron búsquedas en los artículos de Lilacs, BDEnf y Scielo con descriptor de angioplastia, que abordaron las 
complicaciones relacionadas con el procedimiento. Después de la evaluación de las complicaciones, se realizó la inferencia los DE aplicados. Resultados: Se incluyeron trece artículos en el corpus de análisis. Las principales DE deducidas aplicables son: Eliminación urinaria deteriorada; Patrón de respiración ineficaz; Disminución del riesgo de perfusión tisular; Perfusión de tejidos periféricos ineficaz; Riesgo de perfusión tisular cerebral ineficaz; Disminución del gasto cardíaco; Confusión aguda; Integridad de la piel deteriorada; Deterioro de la integridad tisular. Conclusión: Las taxonomías de enfermería permiten las DE aplicables para guiar la sistematización de la asistencia en las complicaciones de los ICP.

Palabras-clave: angioplastia, enfermería cardiovascular, diagnóstico de enfermería, cardiología.

Introdução

As doenças cardiovasculares (DCV) representam uma das maiores causas de mortalidade no mundo e, segundo a Organização Mundial da Saúde (OMS), nos últimos anos, a taxa de mortalidade por DCV variou entre 28 e 34 milhões de óbitos na população mundial, com estimativas de atingir valores superiores a 35 milhões, em 2030 [1].

Os procedimentos de alta complexidade mais utilizados no tratamento das doenças crônicas cardiovasculares são a cirurgia de revascularização miocárdica (RVM) e a angioplastia coronariana (AC), além da colocação de marcapassos permanentes e estudos hemodinâmicos [2].

Na necessidade de confirmar a presença da doença arterial coronariana (DAC), e para determinar a necessidade de tratamento cirúrgico (angioplastia coronariana, cirurgia cardíaca ou correção de cardiopatias congênitas), é indicado o cateterismo cardíaco, também conhecido como cinecoronariografia ou estudo hemodinâmico. Trata-se de um exame invasivo para confirmar a presença de obstruções das artérias coronarianas ou avaliar o funcionamento das válvulas e do músculo cardíaco. Estudos comprovam que os principais preditores de complicação vascular para o procedimento são: sexo feminino, idade superior a 70 anos, diabetes, hipertensão e tabagismo [3-5]. Com o avanço da tecnologia, a angioplastia coronariana $(\mathrm{AC})$ é o método mais utilizado para intervenção coronariana percutânea, tendo em vista a rápida recuperação do paciente [3].

O papel do enfermeiro nos procedimentos hemodinâmicos desenvolve-se em três momentos: pré, trans e pós-procedimento. A orientação, a avaliação e o preparo tanto físico quanto emocional (psicológico) aos pacientes fazem parte do período pré-procedimento. Neste momento, o conhecimento dos temores, dúvidas e expectativas dos pacientes em relação aos exames torna-se fundamental para que o enfermeiro possa assisti-lo de maneira individualizada.

O olhar especializado para a detecção precoce de complicações deve propiciar uma intervenção imediata, tanto nas alterações locais quanto sistêmicas. Além disso, os registros da Sistematização da Assistência de Enfermagem prestada neste caso devem abranger as complicações, mesmo que não tão frequentes no cotidiano, mas sempre que ocorram.

Objetivou-se realizar a inferência de diagnósticos de enfermagem aplicáveis às principais complicações das intervenções coronárias percutâneas nestes casos.

Material e métodos

Trata-se de estudo de revisão integrativa da literatura para a inferência dos Diagnósticos de Enfermagem (DE). A revisão integrativa é um dos métodos utilizados na prática baseada em evidências por permitir a incorporação das evidências na prática clínica. Inclui a análise e síntese de pesquisas relevantes para o apoio à prática clínica e a tomada de decisão [6].

Por propor-se a oferecer o conhecimento atual da literatura sobre determinado tema, a revisão integrativa tem exercido papel fundamental para o aprimoramento dos Diagnósticos de Enfermagem, como etapa inicial do processo de validação [7].

Foram consideradas seis fases no processo de elaboração de uma revisão integrativa: $1^{\text {a }}$ Fase: Identificação do tema ou questionamento da revisão integrativa e elaboração da pergunta norteadora; $2^{2}$ Fase: Amostragem ou busca na literatura; $3^{\underline{a}}$ Fase: Coleta de dados categorização dos estudos; 4 ${ }^{a}$ Fase: Análise crítica dos estudos incluídos na revisão integrativa; 5 Fase: Interpretação dos resultados; 6a Fase: Síntese do conhecimento evidenciado nos artigos analisados ou apresentação da revisão integrativa [6-8]. 
A questão norteadora escolhida foi: quais as principais complicações para os pacientes submetidos a intervenções coronárias percutâneas e possíveis diagnósticos de enfermagem?

A primeira etapa do estudo, desenhada de forma a atender a questão norteadora foi a revisão integrativa. O período de coleta de dados compreendeu o mês de janeiro de 2018. Foram incluídos artigos encontrados na busca nas bases de dados Scielo (Scientific Electronic Library Online), Lilacs (Latin American and Caribbean Center on Health Sciences Information) e BDEnf (Banco de Dados em Enfermagem) com os descritores angioplastia e diagnóstico de enfermagem, resultando em apenas dois resultados no formato de artigo que não contemplavam as possíveis complicações do procedimento. Sendo assim, foi realizada nova busca apenas com o descritor "angioplastia", resultando em 505 artigos com resumos disponíveis. Destes, todos os resumos foram lidos e foram incluídos no corpus de análise artigos que versassem sobre as complicações decorrentes dos procedimentos hemodinâmicos invasivos, resultando em 20 no total.

$\mathrm{Na}$ segunda etapa, a análise dos resultados foi confrontada com a taxonomia de enfermagem NANDA e NIC, no intuito de inferir os diagnósticos de enfermagem e intervenções passíveis de serem utilizadas nessas situações. Para tal, utilizou-se da metodologia de inferência de DE utilizada por Aliti et al. [9], que lança mão do raciocínio clínico e julgamento diagnóstico para inferir DE a partir dos sinais e sintomas identificados com base na experiência clínica e prática no atendimento de pacientes dos pesquisadores [9], utilizando-se a Taxonomia II NANDA-I [10].

\section{Resultados}

Dos 20 artigos utilizados, 13 compuseram o corpus de análise, sendo os outros não selecionados: um por utilizar técnica ulnar, um por apresentar resultados com mais de 30 dias - não sendo mais atendido no laboratório de hemodinâmica, 2 por selecionarem apenas mulheres para o estudo e 3 por não apresentarem a proporção das complicações (Quadro 1).

As principais complicações relatadas foram: óbito $(0,37 \%-4,5 \%)$, infarto do miocárdio periprocedimento $(0,3-4,02 \%)$, acidente vascular cerebral $(0,025-1,81 \%)$, complicações vasculares $(0,37-6,8 \%)$, nefropatia induzida por contraste $(1,27-2,7 \%)$, sangramento $(0,5-$ $5,9 \%)$ e cirurgia de revascularização de emergência/urgência (0,04-0,53\%) (Quadro 1).

Quadro 1 - Registros de complicações pós-intervenção coronária percutânea. Recife/PE, 2017.

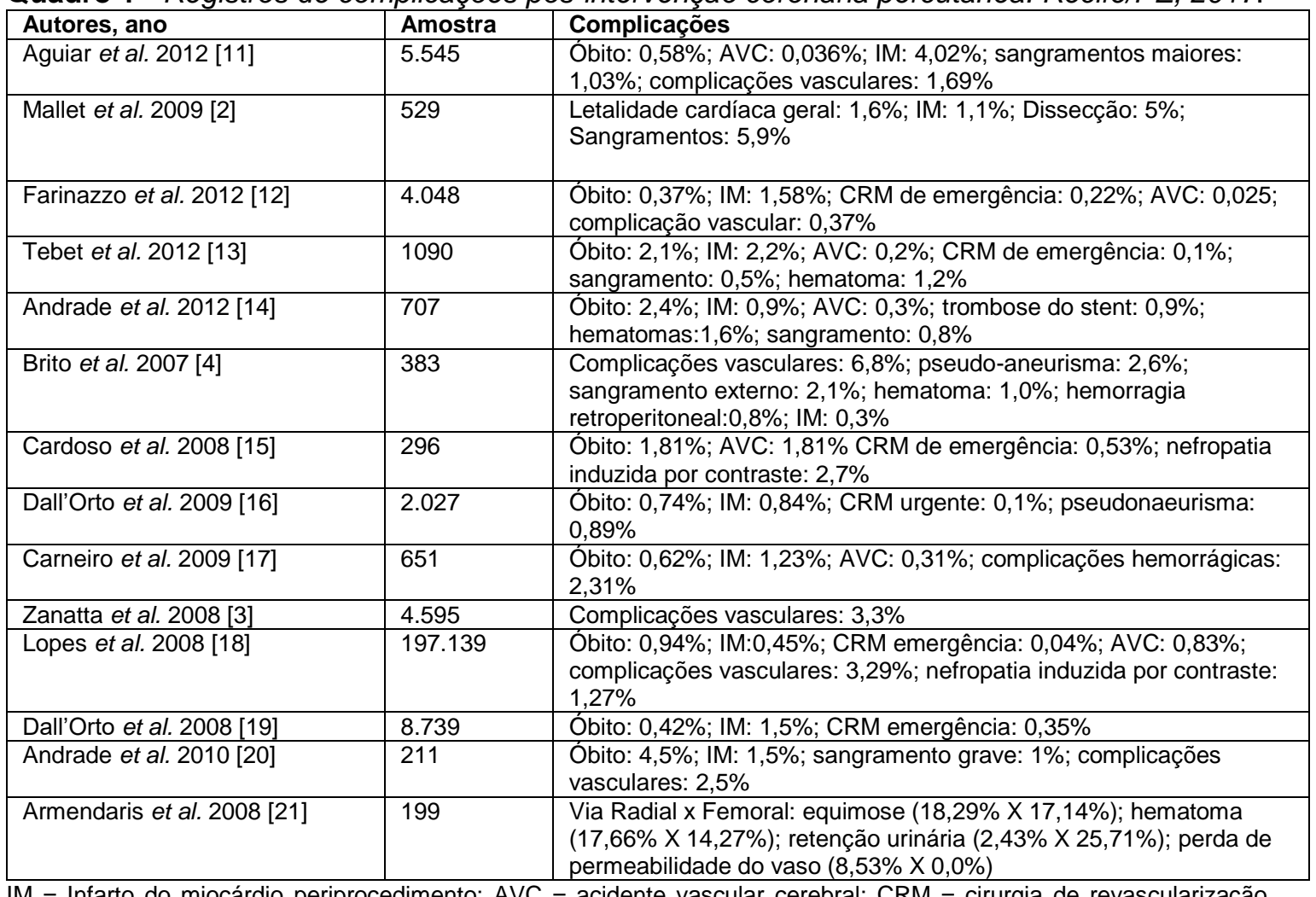

IM = Infarto do miocárdio periprocedimento; AVC = acidente vascular cerebral; CRM = cirurgia de revascularização miocárdica. 


\section{Complicações nas intervenções coronárias percutâneas (ICPS)}

Apesar dos avanços da cardiologia intervencionista, as complicações vasculares permanecem relativamente frequentes, tendo impacto direto na assistência de enfermagem nos serviços de hemodinâmica.

A maior investigação encontrada sobre o tema foi uma publicação a partir dos registros da Central Nacional de Intervenções Cardiovasculares (CENIC), que realizou um estudo com 197.139 intervenções coronárias realizadas no Brasil, que foram registradas entre 1999 até 0 final de 2007, sendo 131.797 (66,85\%) em homens e $65.342(33,15 \%)$ em mulheres [18]. A média de idade foi significativamente superior nas mulheres $(64,0 \pm 11,6$ anos vs. $60,4 \pm 11,7$ anos; $p<0,0001)$. A ICP em mulheres apresenta resultados imediatos discretamente menos satisfatórios que nos homens, em associação à tríade de idade mais avançada, maior prevalência de diabetes melito e menor calibre do vaso [18].

Pesquisa nacional para avaliar a incidência e os preditores contemporâneos de complicações vasculares após intervenções coronárias realizadas por via percutânea femoral, conduzida com 383 pacientes durante a internação hospitalar encontrou um percentual de $6,5 \%$ de complicações vasculares. Destes, o pseudo-aneurisma da artéria femoral foi a complicação mais observada (2,6\%). Os preditores independentes de complicação vascular, neste estudo, foram o sexo feminino e o uso do abciximab [4].

Um grande inquérito avaliando um banco de dados informatizado no total de 4.595 pacientes com 5.485 stents implantados, com média de idade de 60,64 $\pm 10,65$ anos e $32 \%$ de mulheres, as ICP foram realizadas pela via femoral em $95 \%$ dos casos e pela via radial em $5 \%$, e em $85 \%$ dos procedimentos foram utilizados introdutores $6 \mathrm{~F}$ (em $15 \%$ foram usados introdutores $7 F)$. Foram registradas complicações vasculares em $162(3,3 \%)$ pacientes, sendo o único preditor o uso de introdutores $7 F(O R=3,05, \operatorname{IC} 95 \%=1,2-7,8 ; p=0,02)[3]$.

Outra publicação nacional avaliando 529 prontuários de pacientes de angioplastia coronariana realizados no município do Rio de Janeiro, RJ, de 1999 a 2003, encontrou um índice de letalidade cardíaca geral de 1,6\%, variando de $0,9 \%$ a $6,8 \%$ dentre os quatro hospitais estudados, aumentando com a idade. A letalidade na angioplastia coronariana primária e de resgate foi elevada, $17,4 \%$ e 13,1\%, respectivamente; nas angioplastias eletivas foi de $0,8 \%$. As principais complicações foram sangramento $(5,95 \%)$, dissecção (5\%) e oclusão do vaso $(2,6 \%)$ e infarto periprocedimento $(1,1 \%)[2]$.

A intervenção coronária percutânea (ICP) é realizada de forma eletiva ou imediatamente após a cineangiocoronariografia, quando são chamadas de ad hoc. [18]. Uma investigação conduzida com 4.048 pacientes, 1.510 (37,3\%) submetidos à ICP ad hoc e 2.538, a ICP eletiva mostrou que o grupo ICP ad hoc mostrou ser mais jovem, com menor prevalência de comorbidades e maior número de pacientes tratados na vigência da síndrome coronária aguda. Mostraram ter menor gravidade angiográfica, utilizaram stents de maior diâmetro e apresentaram mais distúrbios de fluxo transitórios durante a ICP. O sucesso do procedimento foi semelhante às eletivas, assim como a ocorrência de complicações [17]. Outras publicações confirmaram que a ICP ad hoc é tão segura quanto às eletivas [17].

A idade avançada tem sido um dos preditores de maior sangramento e outras complicações, provavelmente pelo processo de alteração vascular comum ao envelhecimento. Uma pesquisa com o intuito de avaliar os desfechos hospitalares em pacientes com idade superior a 70 anos submetidos à ICP incluiu na análise 296 pacientes (186 com $<70$ anos e $110 \mathrm{com}>70$ anos) e divulgou que não foi observada diferença em relação às características clínico-angiográficas, à necessidade de cirurgia de urgência e de complicações vasculares. Ocorreu tendência a maior mortalidade $(1,81 \%$ vs. $0,53 \% ; p=0,06)$, maior incidência de acidente vascular encefálico $(1,81 \%$ vs. $0 \% ; p=0,06)$, e maior incidência de nefropatia induzida pelo contraste no grupo > 70 anos $(2,7 \%$ vs. $0 \% ; p=0,02)[15]$.

Ainda no tocante à idade, ao se acompanhar 707 pacientes idosos foram submetidos a ICP, dos quais $635(89,8 \%)$ por meio de citado radial, pesquisadores encontraram que a taxa de sucesso angiográfico foi de $96,8 \%$, sendo necessária a troca da via de citado em $2,8 \%$ dos casos [14]. O uso do citado radial associou-se a baixa incidência de sangramento grave, corroborando a indicação desta via de citado [14]. 
Quanto à via de citado e suas relações com as complicações, um estudo nacional realizado com 5.545 pacientes submetidos a intervenções coronárias percutâneas, tradicionalmente realizadas pela via femoral, dos 298 dos procedimentos realizados por via arterial, avaliou-se que o perfil clínico era menos complexo quando indicada essa via e que não houve diferenças nos desfechos combinados [15]. No entanto, foi observada menor incidência de sangramentos maiores $(0,4 \%$ vs. $1,3 \% ; P<0,01)$ e de complicações vasculares $(0,5 \%$ vs. $2,2 \% ; \mathrm{P}<0,01)$ incluindo hematomas $>5 \mathrm{~cm}$, pseudoaneurismas e fístulas arteriovenosas ou hemorragias com necessidade de transfusão ou cirurgia [11].

A via radial vem sendo utilizada de forma crescente em procedimentos diagnósticos e terapêuticos, por possuir diversas vantagens, como comodidade para o paciente no pósprocedimento imediato, diminuição do tempo de internação com consequente redução dos custos hospitalares e menor índice de complicações no sítio de punção, quando comparada à via femoral. [13,20,22].

Um estudo apenas com ICP de citado radial a partir do registro prospectivo de 1.117 pacientes que realizaram ICP, a sedação foi realizada em $58,5 \%$ dos pacientes e obteve-se sucesso angiográfico de $96,2 \%$. Neste estudo, a utilização do citado radial na ICP mostrou alto índice de sucesso e baixo índice de eventos cardíacos maiores e de complicações hemorrágicas [13].

Outro trabalho, no qual foi realizada análise retrospectiva de 2.027 pacientes consecutivos submetidos à intervenção coronária percutânea eletiva, a via radial foi realizada em $27 \%$ dos pacientes, dos quais $45,1 \%$ apresentavam lesões uniarteriais $(45,1 \%$ vs. $37,3 \%$; $P$ $<0,001)$, apresentaram maior sucesso ( $99 \%$ vs. $97,3 \%$; $P=0,046)$, menor número de eventos cardíacos adversos maiores nas fases tanto hospitalar $(0,7 \%$ vs. $2 \% ; P=0,043)$ como tardia $(11,3 \%$ vs. $16,3 \% ; P<0,005)$ em relação a via femoral [16].

Em um dos artigos foi apresentada e utilizada a classificação do estudo Early Discharge after Transradial Stenting of Coronary Arteries (EASY) para hematomas de citados radiais: tipo I, $<5 \mathrm{~cm}$ de diâmetro; tipo II, $<10 \mathrm{~cm}$ de diâmetro; tipo III, $>10 \mathrm{~cm}$, sem atingir 0 cotovelo; tipo IV, hematoma estendendo-se além do cotovelo; tipo $\mathrm{V}$, qualquer hematoma com injúria isquêmica à mão [13]. Complicações relacionadas ao sítio de punção, além de hematomas, incluíram fístula arteriovenosa, pseudoaneurisma, oclusão arterial assintomática, necessidade de reparo vascular cirúrgico e infecção local [17].

\section{Inferência dos diagnósticos de enfermagem relacionados às ICP}

A enfermagem deve estar preparada para a assistência ao paciente no laboratório de hemodinâmica, e em particular, atenta aos sinais de possíveis complicações que possam apresentar. Contudo, apesar da relevância, não foi encontrado estudo nacional que tivesse por objeto a aplicação dos Diagnósticos de Enfermagem para as complicações das ICP.

Em estudo recente, foi realizado um levantamento dos diagnósticos de enfermagem presentes em 233 pacientes atendidos em um Laboratório de Hemodinâmica. Para fins de discussão, foram considerados diagnósticos de enfermagem que apresentaram frequência acima do percentil 30: risco de infecção; risco de sangramento; comunicação verbal prejudicada; risco de intolerância a atividade; dor aguda; risco de perfusão tissular cardíaca diminuída; déficit no autocuidado para banho; ansiedade; medo; integridade da pele prejudicada e conforto prejudicado [23]. Apesar de questionar-se o julgamento de risco para 0 título Intolerância à atividade, visto que os pacientes a apresentam no mais das vezes na isquemia miocárdica e após os procedimentos pode-se dizer empiricamente, que diagnósticos citados na referência são dos mais utilizados na Sistematização da Assistência de Enfermagem a esses pacientes.

Depois da avaliação da literatura na primeira etapa deste estudo, os pesquisadores passaram a elaborar, a partir destes resultados, a inferência dos diagnósticos de enfermagem. O quadro 2 apresenta os DE elencados a partir das complicações encontradas na etapa da revisão. Os DE foram elaborados a partir da taxonomia II da NANDA-I e são apresentados com os referidos códigos, características definidoras e fatores relacionados [10].

Os principais diagnósticos aplicáveis elaborados são:

- Relacionado à nefropatia induzida por contraste: Eliminação urinária prejudicada (00016);

- Relacionado ao infarto periprocedimento: Risco de perfusão tissular cardíaca diminuída (00200); Padrão respiratório ineficaz (00032); Débito cardíaco diminuído (00029); 
- Relacionados ao acidente vascular cerebral: Perfusão tissular periférica ineficaz (00204); Risco de perfusão tissular cerebral ineficaz (00201); Confusão aguda (00128);

- Relacionado às complicações vasculares locais e sangramentos: Integridade da pele prejudicada (00046); Integridade tissular prejudicada (00044).

Quadro 2 - Inferência dos Diagnósticos de Enfermagem aplicáveis às principais complicações das intervenções coronárias percutâneas. Recife/PE, 2017.

\begin{tabular}{|l|l|l|}
\hline Diagnóstico de Enfermagem & Característica Definidora & Fatores Relacionados \\
\hline $\begin{array}{l}\text { Eliminação urinária prejudicada } \\
\text { (00016) }\end{array}$ & Frequência diminuída & $\begin{array}{l}\text { Múltiplas causas (nefropatia } \\
\text { induzida por contraste) }\end{array}$ \\
\hline Padrão respiratório ineficaz (00032) & $\begin{array}{l}\text { Dispneia, taquipneia, uso da } \\
\text { musculatura acessória }\end{array}$ & Dano neurológico (AVC) \\
\hline $\begin{array}{l}\text { Risco de perfusão tissular cardíaca } \\
\text { diminuída (00200) }\end{array}$ & Não se aplica & Espasmo da artéria coronariana \\
\hline $\begin{array}{l}\text { Perfusão tissular periférica ineficaz } \\
\text { (00204) }\end{array}$ & $\begin{array}{l}\text { Parestesia, pulsos ausentes ou } \\
\text { diminuídos, dor em extremidade, } \\
\text { edema, Tempo de enchimento } \\
\text { capilar }>\text { 3seg, claudicação }\end{array}$ & $\begin{array}{l}\text { Hipertensão, diabetes, } \\
\text { sedentarismo, tabagismo }\end{array}$ \\
\hline $\begin{array}{l}\text { Risco de perfusão tissular cerebral } \\
\text { ineficaz (00201) }\end{array}$ & Não se aplica & $\begin{array}{l}\text { Aterosclerose aórtica, dissecção } \\
\text { arterial, embolia, efeitos } \\
\text { secundários relativos ao } \\
\text { tratamento (angioplastia), } \\
\text { terapia trombolítica }\end{array}$ \\
\hline Débito cardíaco diminuído (00029) & $\begin{array}{l}\text { Alterações no ECG, arritmias, } \\
\text { palpitações, dispneia, pele fria e } \\
\text { pegajosa, pulsos periféricos } \\
\text { diminuídos }\end{array}$ & $\begin{array}{l}\text { Contratilidade, frequência } \\
\text { cardíaca, pós e pré-carga, ritmo } \\
\text { e/ou volume de ejeção alterados }\end{array}$ \\
\hline Confusão aguda (00128) & $\begin{array}{l}\text { Agitação aumentada, flutuação } \\
\text { na atividade psicomotora e no } \\
\text { nível de consciência }\end{array}$ & Delirium (AVC) \\
\hline $\begin{array}{l}\text { Integridade da pele prejudicada } \\
\text { (00046) }\end{array}$ & $\begin{array}{l}\text { Destruição de camadas da pele, } \\
\text { invasão de estruturas do corpo, } \\
\text { rompimento da superfície da pele } \\
\text { (hematomas) }\end{array}$ & $\begin{array}{l}\text { Fatores mecânicos } \\
\text { (introdutores, cateteres, etc.) }\end{array}$ \\
\hline $\begin{array}{l}\text { Integridade tissular prejudicada } \\
\text { (00044) }\end{array}$ & $\begin{array}{l}\text { Tecido subcutâneo destruído ou } \\
\text { lesado (equimose, complicações } \\
\text { no local de punção) }\end{array}$ & $\begin{array}{l}\text { Fatores mecânicos } \\
\text { (introdutores, cateteres, etc.) }\end{array}$ \\
\hline
\end{tabular}

O enfermeiro deve estar atento aos sinais de alerta e aos riscos de complicações, monitorizando a beira do leito com muita atenção todo paciente após procedimentos hemodinâmicos invasivos, antes, durante e após a retirada do introdutor, até a transferência da mesa do procedimento para o repouso/recuperação. O reconhecimento precoce pode minimizar o dano causado pelas complicações.

O exercício da inferência dos Diagnósticos de Enfermagem a partir da literatura pertinente às complicações destes procedimentos revelou nove DE prioritários para essas pacientes nestas situações, sendo dois deles diagnósticos de risco. A partir desses achados, os enfermeiros podem pensar intervenções e estratégias de ação para protocolos em seus serviços.

Por fim, vale ressaltar que os diagnósticos de enfermagem possíveis de serem aplicados nestes casos de eventos não esperados, devem ser passíveis de registro nos instrumentos destinados a documentação da Sistematização da Assistência de Enfermagem prestada, impresso ou em meio eletrônico.

1. Freitas EO, Nogueira RS, Stekel LMC, Bublitz S, Kirchof R, Guido LA. Perfil dos pacientes com doença arterial coronariana submetidos ao cateterismo cardíaco. Rev Enferm UFSM 2013;3(Esp):679-688. https://doi.org/10.5902/2179769211124 
2. Mallet ALR, Oliveira GMM, Klein $\mathrm{CH}$, Carvalho MRM, Souza e Silva NA. Letalidade e complicações de angioplastias em hospitais públicos no Rio de Janeiro, RJ. Rev Saúde Pública 2009;43(6):917-27. https://doi.org/10.1590/s0034-89102009005000078

3. Zanatta LG, Cardoso CO, Mota FM, Conti EP, Diehl D, Rodrigues APR et al. Preditores e incidência de complicações vasculares após a realização de intervenções coronárias percutâneas: achados do registro IC-FUC. Rev Bras Cardiol Invasiva 2008;16(3):301-6 https://doi.org/10.1590/s2179-83972008000300010

4. Brito Junior FS, Magalhães MA, Nascimento TCDC, Amorim IMG, Almeida BO, Abizaid A, Perin MA. Incidência e preditores contemporâneos de complicações vasculares após intervenção coronária percutânea. Rev Bras Cardiol Invasiva 2007;15(4):394-9. https://doi.org/10.1590/s2179-83972007000400014

5. Carvalho MS, Calé R, Gonçalves PA, Vinhas H, Raposo L, Teles R, Martins $\mathrm{C}$ et al. Fatores preditivos da conversão de acesso radial em femoral no cateterismo cardíaco. Arq Bras Cardiol 2015;104(5):401-8. https://doi.org/10.5935/abc.20150017

6. Mendes KDS, Silveira RCCP, Galvão CM. Revisão integrativa: método de pesquisa para a incorporação de evidências na saúde e na enfermagem. Texto Contexto Enferm 2008;17(4):758-64. https://doi.org/10.1590/s0104-07072008000400018

7. Pompeo DA, Rossi LA, Galvão CM. Revisão integrativa: etapa inicial do processo de validação de diagnóstico de enfermagem. Acta Paul Enferm 2009;22(4):434-8. https://doi.org/10.1590/s0103-21002009000400014

8. Polit DF, Beck CT, Hungler BP. Fundamentos de pesquisa em enfermagem: métodos, avaliação e utilização. Porto Alegre: Artmed; 2004.

9. Aliti GB, Linhares JCC, Linch GFC, Ruschel KB, Rabelo ER. Sinais e sintomas de pacientes com insuficiência cardíaca descompensada: inferência dos diagnósticos de enfermagem prioritários. Rev Gaúcha Enferm 2011;32(3):590-5. https://doi.org/10.1590/s1983-14472011000300022

10. NANDA-I. Diagnósticos de enfermagem da NANDA: definições e classificação 20152017. Porto Alegre: Artmed; 2015.

11. Lopes MACQ, Barros MAV, Oliveira IR, Martins HC, Paiva MS, Lima JAC et al. Comparação do perfil epidemiológico, clínico e dos resultados das intervenções coronárias percutâneas entre os gêneros masculino e feminino, na população brasileira: dados do registro CENIC. Rev Bras Cardiol Invasiva 2008;16(4):463-73. https://doi.org/10.1590/s2179-83972008000400017

12. Farinazzo MM, Cantarelli MJC, Castello Jr HJ, Gonçalves R, Gioppato S, Guimarães JBF et al. Resultados Hospitalares da Intervenção Coronária Percutânea Ad Hoc vs. Eletiva. Rev Bras Cardiol Invasiva 2012;20(4):373-8. https://doi.org/10.1590/s217983972012000400006

13. Carneiro JKR, Carneiro Neto JD, Lima Neto JA, Parente FL, Aguiar LS, Rocha BAM et al. Segurança e eficácia da intervenção coronária percutânea ad hoc em pacientes com angina estável. Rev Bras Cardiol Invasiva 2009;17(2):196-201. https://doi.org/10.1590/s2179-83972009000200011

14. Cardoso CO, Cardoso CR, Rodrigues LHC, Staudt C, Duarte AFS, Miller VM et al. Intervenção coronariana percutânea em pacientes com mais de 70 anos: Análise de desfechos intra-hospitalares na era contemporânea. Rev Bras Cardiol Invasiva 2008;16(1):64-9. https://doi.org/10.1590/s2179-83972008000100013

15. Andrade PB, Tebet MA, Andrade MVA, Barbosa RA, Mattos LA, Labrunie A. Impacto da utilização do citado radial na ocorrência de sangramento grave entre idosos submetidos à Intervenção Coronária Percutânea. Rev Bras Cardiol Invasiva 2012;20(1):16-20. https://doi.org/10.1590/s2179-83972012000100005

16. Aguiar Filho GB, Siqueira DA, Chaves AJ, Coelho FM, Costa Junior JR, Veloso M et al. Intervenção coronária percutânea pela via radial: incorporação da técnica e resultados de um centro de formação em cardiologia intervencionista. Rev Bras Cardiol Invasiva 2012;20(4):367-72. https://doi.org/10.1590/s2179-83972012000400005

17. Tebet MA, Andrade PB, Nogueira EF, Esteves V, Matos MPB, Andrade MVA et al. Características das ICPs em centro que prioriza a utilização do acesso radial. Rev Bras Cardiol Invasiva 2012;20(3):288-94. https://doi.org/10.1590/s2179-83972012000300012

18. Andrade PB, Tebet MA, Silva FSM, Andrade MVA, Mattos LA, Labrunie A. Utilização do citado radial elimina ocorrência de sangramento grave relacionado ao sítio de punção após intervenção coronariana percutânea primária. Rev Bras Cardiol Invasiva 2010;18(4):387-91. https://doi.org/10.1590/s2179-83972010000400006 
19. Boechat e Salles JA, Andrea JCM, Cortes LA, Carestiato LV, Santos LFC, Figueira HRL. Análise comparativa de segurança e eficácia entre as vias de citado radial e femoral na realização de intervenção coronária percutânea no infarto agudo do miocárdio. Rev Bras Cardiol Invasiva 2009;17(4):498-504. https://doi.org/10.1590/s2179-83972009000400013

20. Dall'Orto CC, Lapa GA, Carnieto NM, Siqueira B, Oliveira Neto JB, Mauro MFZ, et al. Experiência inicial utilizando a via radial no tratamento percutâneo de doença coronária. Rev Bras Cardiol Invasiva 2009;17(2):214-9. https://doi.org/10.1590/s217983972009000200014

21. Oliveira MF, Silva LF. Enfermagem em laboratório de hemodinâmica: diagnóstico e intervenção fundamentados na Teoria da Adaptação de Roy. Rev Eletr Enf 2010;12(4):678-85. https://doi.org/10.5216/ree.v12i4.8325 